\title{
Fulfilling the Promise of Hospital Medicine: Tailoring Internal Medicine Training to Address Hospitalists' Needs Tracking \#5134r3
}

\author{
Jeffrey J. Glasheen, $M D^{1,2,3}$, Eric M. Siegal, $M D^{4}$, Kenneth Epstein, $M D^{5}$, Jean Kutner, $M D^{2}$, \\ and Allan V. Prochazka, MD
}

IInternal Medicine Residency Training Program, University of Colorado Denver School of Medicine, Aurora, CO, USA; ${ }^{2}$ Division of General Internal Medicine, Department of Medicine, University of Colorado Denver School of Medicine, Aurora, CO, USA; ${ }^{3} \mathrm{Hospital}$ Medicine Service, University of Colorado Denver School of Medicine, Aurora, CO, USA; ${ }^{4}$ Cogent Healthcare, University of Wisconsin School of Medicine and Public Health, Madison, WI, USA; ${ }^{5}$ Medical Affairs and Clinical Research, IPC-The Hospitalist Company, University of Colorado Denver School of Medicine, Aurora, CO, USA; 'Denver VA Medical Center, University of Colorado Denver School of Medicine, Aurora, CO, USA.

Categorical internal medicine (IM) residency training has historically effectively prepared graduates to manage the medical needs of acutely ill adults. The development of the field of hospital medicine, however, has resulted in hospitalists filling clinical niches that have been traditionally ignored or underemphasized in categorical IM training. Furthermore, hospitalists are increasingly leading inpatient safety, quality and efficiency initiatives that require understanding of hospital systems, multidisciplinary care and inpatient quality assessment and performance improvement. Taken in this context, many graduating IM residents are under-prepared to practice as effective hospitalists. In this paper, we outline the rationale for targeted training in hospital medicine and discuss the content and methods for delivering this training.

KEY WORDS: medical education; hospitalist training; hospitalist; hospital medicine; residency redesign.

$\mathrm{J}$ Gen Intern Med 23(7):1110-5

DOI: $10.1007 / \mathrm{s} 11606-008-0646-5$

(C) Society of General Internal Medicine 2008

\section{BACKGROUND}

Hospital medicine is the fastest-growing medical discipline in the United States. There are now over 20,000 practicing hospitalists, with a potential workforce approaching 3040,000 , surpassing cardiology and emergency medicine in size. $^{1,2}$ More than $80 \%$ of American hospitalists are internists. ${ }^{3}$ The expansion of hospitalists' numbers has been paralleled by an expansion of their job descriptions-requiring new skills that are generally not taught in most categorical internal medicine (IM) training programs.

Historically, IM training has been an excellent medium for training hospital-based clinicians. Categorical IM training programs emphasize inpatient ward and intensive care experiences, and graduates are generally well prepared to manage

This paper has not been presented at any conferences. This work was not funded by a grant. most inpatient medical conditions. ${ }^{4}$ During the early days of hospital medicine, this scope of training was probably adequate as hospitalists focused primarily upon the clinical care of patients who had been traditionally cared for by general internists. The first hospitalists were frequently members of group practices who either rotated between the hospital and clinic or spent most, if not all of their time, caring for their practices' medical inpatients. ${ }^{5-7}$ Similarly, the first academic hospitalists simply replaced general internists and subspecialists as attending physicians on general medical ward teams. ${ }^{8}$

In addition to mastering clinical skills, IM-trained hospitalists will increasingly be expected to provide multidisciplinary care, integrate information technology into daily workflow and adopt rigorous quality assessment and improvement methodology to deliver state-of-the-art care. While it would be ideal for all residents to master this expanding repertoire of competencies, work hour restrictions have limited available time in training. As such, 3 years of post-graduate training will become increasingly insufficient to produce Oslerian generalists, able to care for patients with diverse disease states in all settings. In response, IM professional societies have recently published position papers calling for universal training in core IM supplemented by specialized offerings tailored to a resident's future professional needs. ${ }^{9-11}$ In this paper, we outline the rationale for targeted training in hospital medicine and discuss the content that would define this training.

\section{Current Hospitalist Practice}

From the beginning, hospitalists were expected to demonstrate value by improving outcomes, managing hospital throughput, reducing length of stay and decreasing the overall cost of care. Hospitalists were not explicitly trained to accomplish these goals; rather their success was a byproduct of their increased inpatient clinical experience and greater presence in the hospital. ${ }^{12,13}$ Over time, however, hospitalists accepted expanded clinical and administrative roles that fell outside the boundaries of traditional IM residency training. ${ }^{14-16}$ Eighty-six percent of hospital medicine groups now participate in quality improvement (QI) initiatives, and $72 \%$ contribute to the development of practice guidelines. ${ }^{3}$ Fifty-nine percent partake in formal utilization review, and 54\% are involved in hospital electronic medical record and computer provider order entry initiatives. ${ }^{3}$ Hospitalists also commonly lead 
efforts to improve patient handoffs, partner with outpatient providers in the care of complex medical patients and spearhead inter-disciplinary rounds in their hospitals. ${ }^{17-21}$ Finally, hospitalists are developing hospital-based services such as palliative care and rapid response teams. ${ }^{22}$

Hospitalists' clinical roles have also changed. While internists have long provided consultation to medically complex patients admitted to other services, hospitalists are now assuming significant, if not primary, responsibility for the care of "nonmedical" patients. ${ }^{23-27}$ This marks a significant departure from traditional consultative medicine, where the consultant is expected to answer a specific question and offer recommendations. $^{28}$ The new paradigm of medical co-management, which unites a hospitalist with an attending from another specialty in the care of a patient, frequently places the hospitalist in the role of primary attending and mandates an approach to communication, responsibility-sharing and management with other professionals that is rarely modeled or taught in many teaching programs.

Hospitalists may also be under-prepared to manage the clinical demands of patients with non-medical diagnoses. As the attending physician for a patient with a hip fracture, the hospitalist may not only be responsible for acute and chronic medical issues, but also for the perioperative risk assessment and the management of postoperative anemia, pain control (including epidural and spinal anesthesia) and anticoagulation. The hospitalist may be asked to educate families and patients regarding the surgery itself, manage the care transition and recommend activity, rehabilitation and post-discharge follow-up. Many hospitalists have further extended their co-management roles in perioperative care to the preoperative arena. ${ }^{29}$ These evolutionary changes represent a major shift in clinical responsibility for hospitalists, yet traditional IM training programs rarely teach these skills.

\section{The Case for Hospitalist-Focused Training}

In a survey of 389 IM-trained hospitalists, significant educational mismatches, defined as skills important to current practice but underemphasized in residency training, were prevalent in systems issues (e.g., QI, health-care finance, utilization review, practice guideline development), continuums of care (e.g., coordination of care between settings, prescribing the appropriate level of post-discharge care) and ethics (e.g., palliative care, principles of advance directives). Respondents also noted that their residency training underemphasized clinical areas such as geriatrics, neurology and perioperative and consultative medicine, while overemphasizing the medical procedures needed for the practice of hospital medicine. ${ }^{4}$

We recently described the experience of 436 community hospitalists employed by a national hospitalist company that staffed 133 hospitals in 11 major US cities. ${ }^{23}$ Nearly $30 \%$ of the patients were seen in a consultative role $(6.4 \%)$ or had neurologic $(13.4 \%)$, orthopedic $(6.4 \%)$ or general surgical $(2.2 \%)$ diagnoses. Forty-six percent of the patients were geriatric, with the largest subgroup of patients (18.8\%) being 75-84 years old. All of these competencies were noted by Plauth et al. as areas of relative undertraining for hospitalists. ${ }^{4}$

Published analyses of the case mix of residents in traditional IM training programs are not readily available. However, we can gain insight by examining published data from academic hospitalist groups, which are closely interwoven into IM training programs and likely reflect the overall case mix in the training program. Of the seven publications from academic hospitalist groups that included substantial case mix information, three described only a single neurologic diagnosis, and none listed an orthopedic condition in their 15 most common diagnoses. ${ }^{8,12,13,30-33}$ Only one of the programs that recorded a neurologic diagnosis was based at a university hospital. ${ }^{32}$ In comparison, our study showed that orthopedic (hip fracture) and neurologic diagnoses (syncope, acute stroke, seizure and transient ischemic attack) comprised a third of the top 15 diagnoses that hospitalists manage.

While all IM trainees must acquire basic competency in QI and patient safety, the level and type of expertise required to effectively practice hospital medicine may differ significantly from that required for traditional outpatient or subspecialty medicine. ${ }^{34}$ Hospitalists who lead hospital-wide QI initiatives must engage complex systems with multiple stakeholders to drive clinical and operational improvements. These initiatives often reach patients who are rarely if ever seen by hospitalists, such as women on a labor and delivery unit, or patients in a burn unit. Thus, to effectively direct QI initiatives, hospitalists must not only be change agents for their own practices, but for the entire hospital system. Hospitals, under increasing scrutiny and accountability for their clinical outcomes, will expect hospitalists to be fully prepared to effectively lead and manage major performance improvement initiatives. These expectations will only grow as public accountability and pay for performance alter the health-care landscape in coming years.

To meet these expectations, hospitalists must possess competency in health system management to promote a performance improvement agenda and the project management tools to deliver meaningful results. To succeed, hospitalists will need skills that most graduating IM residents do not possess. Hospital medicine directors will also have to develop the negotiation skills necessary to effectively make the case to those funding their programs to merit value over productivity by allocating time and resources for quality, patient safety and systems improvement work.

\section{Developing Hospitalist-Focused Training}

Developing curricula to address hospitalist-focused training is a complex process entwining national workforce trends, institutional funding and the need to broadly train IM residents. The Society of Hospital Medicine (SHM) has defined and published The Core Competencies for Hospital Medicine, and we recommend that teaching institutions evaluate their own curricula against this benchmark. ${ }^{35,36}$ While some programs might choose to expose all of their residents to the entire hospital medicine curriculum, we believe this approach negates the benefits of tailoring training to residents' future practice environments and risks overloading an already packed curriculum.

Hospitalist-specific training could be structured in a variety of ways, including additional "mastery" years of residency training (i.e., 4th year), clinical hospital medicine fellowships or tailored training within the current structure of IM residency programs. ${ }^{37,38}$ While all of these options merit further exploration, we believe redesigning IM residency to support tailored vocational offerings in the latter years of training will best achieve this goal. This model could also be used as a template for focused training in other areas of IM such as women's health, HIV or comprehensive ambulatory medicine. 


\section{Guiding Principles in Designing Hospitalist- Focused Training (Table 2)}

Maintain Balance. Internal medicine training must be redesigned in the context of limited resident work hours, declining interest in generalist careers and competing interests for residents' time. Successful programs must balance hospitalist training with the need to broadly train generalist providers, ensuring strong core subspecialty and outpatient medicine training. Hospitalist-focused training cannot entirely abandon ambulatory medicine, as not all trainees who matriculate through the track will ultimately practice exclusively in the hospital. Even full-time hospitalists will need to understand enough ambulatory care to effectively and safely transition patients to and from the hospital.

Achieving this balance will require frank assessments of programmatic service needs versus the educational needs of trainees and commitments from departmental and residency leadership to reallocate resident resources as necessary. Many academic programs have already replaced some or most of their traditional ward teaching faculty with hospitalists. While this is a necessary first step to crafting hospital medicine teaching experiences, it is only a first step. Hospitalist teaching services must also model the workflow, values and integration of care that are central to the practice of hospital medicine. For example, hospitalist ward teams might add co-management of surgical patients into daily workflow, integrate housestaff into daily multidisciplinary rounds or admit new patients every day rather than on a staggered schedule.

Build Operational Improvement Methodology Into Clinical Curricula. Hospital medicine is predicated upon the assumption that hospitalists will improve the efficiency and effectiveness of inpatient care through process redesign and QI. Unfortunately, relatively few practicing hospitalists have the skills necessary to develop and deliver system changes of this magnitude. Matriculating hospitalists with these skills will require programmatic commitment to integrating patient safety, QI and resource utilization curricula into inpatient didactic, clinical and project-based opportunities. Hospital medicine trainees should identify an existing hospital quality or operational deficit and negotiate, develop and implement improvement processes. Completion of such a project should be mandatory for all hospitalist trainees.

Emphasize Process Over Clinical Content. IM housestaff have ample opportunity to learn clinical medicine and pathophysiology throughout their training. Hospitalist-specific rotations should therefore emphasize process issues that are not typically taught on traditional ward rotations, such as standardization of care, continuous QI, transitions of care and resource utilization. Hospitalist trainees should fully participate in multidisciplinary rounds involving pharmacists, nurses, case managers and other members of the care team. They should be taught how to communicate effectively and efficiently with the emergency department, consultants and hospital staff. Successful hospitalist-based training must spotlight the importance of and methods to improve communication and care transitions.
Keep the Tent Big. Hospitalist-focused training cannot occur in a vacuum, and it is important to recognize that restructuring inpatient training will require input and support from other specialties. Hospitalist training programs will depend upon other specialties to provide clinical training opportunities, such as orthopedic co-management or acute stroke care. Many will also likely depend upon non-hospitalist educators to teach disciplines such as geriatrics, palliative care or $\mathrm{BI}$ methodology. Furthermore, including non-hospitalist faculty in curriculum development and teaching recognizes that hospital medicine training probably will not be exclusive to future hospitalists. Housestaff who intend to pursue fellowship training in cardiology, critical care and other hospital-focused specialties will likely choose hospitalist training programs as well. Finally, we believe that all IM housestaff, irrespective of their future career paths, should be able to sample aspects of this training as they deem appropriate.

\section{Framework for Developing Hospitalist-Focused Training}

Programs should begin by performing a needs assessment that compares their current educational environments to the criteria outlined in SHM's Core Competencies for Hospital Medicine as well as other previously underemphasized areas of training (Tables 1, 2 and 3). We do not anticipate uniform findings across programs; for example, residents matriculating from community IM training programs, which do not compete with neurology services for cases, may already receive excellent training in acute stroke care. Programs will need to tailor their solutions to address their specific educational deficits consistent with their local resources, culture and educational philosophies.

These solutions should be developed in the context of the Accreditation Council for Graduate Medical Education's (ACGME) six core competencies. Most of these deficiencies will touch multiple domains. However, in our experience many of the underemphasized areas will fall into the patient care (e.g., acute end-of-life pain and symptom management), medical knowledge (e.g., blood pressure management in acute stroke), systems-based practice (e.g., inter-disciplinary protocol development) and practice-based learning and improvement (e.g., developing and leading QI projects) competencies.

After completing a needs assessment, programs must assess their institutions' level of commitment to hospitalistfocused training and generate broad buy-in. We recommend beginning by engaging local thought leaders and generating their support. It will take commitment and persistence to dispel the dogma that current IM training programs are sufficient to develop fully competent hospitalists. Early in the process, programs should open discussions regarding the impact of educational change on clinical service needs. This often presents a formidable hurdle to overcome, and in many cases success will require coordinated support from both departmental and hospital leadership. IM program directors, departmental chairs and hospital administrators often have widely disparate agendas for their hospitalist programs. Program directors may view hospitalists as a mechanism to comply with ACGME work load restrictions, department chairs may believe that they are a means to expand or create new clinical service lines, and hospital administrators may see an opportunity to improve operational efficiency and quality. The 
Table 1. Underemphasized Areas of Training for Hospitalists*

\begin{tabular}{|c|c|}
\hline Clinical areas & Examples of training needs \\
\hline $\begin{array}{l}\text { Perioperative } \\
\text { medicine }\end{array}$ & $\begin{array}{l}\text { Surgical risk assessment and patient } \\
\text { optimization } \\
\text { Perioperative pain and symptom management } \\
\text { Perioperative venous thromboprophylaxis } \\
\text { Perioperative diabetes management } \\
\text { Rapid assessment of the surgical abdomen }\end{array}$ \\
\hline $\begin{array}{l}\text { Consultative } \\
\text { medicine }\end{array}$ & $\begin{array}{l}\text { Effective medical consultation } \\
\text { Understanding the consultant versus co- } \\
\text { management role }\end{array}$ \\
\hline Orthopedics & $\begin{array}{l}\text { Management of acute hip fracture and joint } \\
\text { arthroplasty } \\
\text { Basics of acute rehabilitation medicine }\end{array}$ \\
\hline $\begin{array}{l}\text { Neurology/ } \\
\text { neurosurgery }\end{array}$ & $\begin{array}{l}\text { Management of blood pressure and diabetes } \\
\text { in acute stroke } \\
\text { Indications and contraindications to } \\
\text { thrombolytic use in acute stroke } \\
\text { Risk assessment in transient ischemic attack } \\
\text { Management of acute seizure and status } \\
\text { epilepticus } \\
\text { Acute medical management of intracranial, } \\
\text { subarachnoid and subdural hemorrhage }\end{array}$ \\
\hline Geriatrics & $\begin{array}{l}\text { Rational medication withdrawal in the acute } \\
\text { care setting } \\
\text { Delirium, fall and pressure ulcer recognition, } \\
\text { prevention and treatment } \\
\text { Preventing in-hospital functional and } \\
\text { nutritional decline }\end{array}$ \\
\hline Palliative care & $\begin{array}{l}\text { Acute pain and symptom management } \\
\text { Understanding the Medicare hospice benefit } \\
\text { Feeding tube use at the end of life }\end{array}$ \\
\hline Non-clinical areas & Examples of training needs \\
\hline $\begin{array}{l}\text { Health-care } \\
\text { economics }\end{array}$ & $\begin{array}{l}\text { Understanding the stakeholders whose } \\
\text { decisions drive hospital policy } \\
\text { Understanding hospital reimbursement as a } \\
\text { driver of hospitalist compensation and } \\
\text { performance } \\
\text { Understanding hospital utilization review }\end{array}$ \\
\hline $\begin{array}{l}\text { Patient safety and } \\
\text { quality } \\
\text { improvement }\end{array}$ & $\begin{array}{l}\text { Common inpatient safety problems and methods } \\
\text { for analysis (e.g., root cause analysis) } \\
\text { Role of human factors and hospital systems in } \\
\text { adverse events } \\
\text { Developing and leading systemic QI and } \\
\text { patient safety initiatives } \\
\text { Practice guideline/protocol development and use } \\
\text { Hospital information systems development, } \\
\text { implementation and management }\end{array}$ \\
\hline $\begin{array}{l}\text { Practice } \\
\text { management }\end{array}$ & $\begin{array}{l}\text { Understanding hospitalist employment } \\
\text { contracts, terms and conditions } \\
\text { Inpatient billing and coding } \\
\text { Risk management and malpractice for the } \\
\text { hospitalist }\end{array}$ \\
\hline Continuums of care & $\begin{array}{l}\text { Transitions of care between patient settings } \\
\text { Key elements of an effective patient hand-off } \\
\text { Prescribing the appropriate level and type of } \\
\text { post-discharge care }\end{array}$ \\
\hline Leadership skills & $\begin{array}{l}\text { Methods to effect organizational change } \\
\text { Negotiation and conflict resolution skills } \\
\text { Importance of personal influence as a } \\
\text { management tool to achieve goals } \\
\text { Demonstrating the value of QI and patient safety } \\
\text { work }\end{array}$ \\
\hline Communication & $\begin{array}{l}\text { Appropriate and timely communication with } \\
\text { referring practioners } \\
\text { Key elements of an effective patient hand off } \\
\text { Facilitating family meetings } \\
\text { Delivering bad news effectively } \\
\text { Leading and participating in a } \\
\text { multidisciplinary team approach to care } \\
\text { Disclosure of medical errors to patients } \\
\text { Determining patient competence and capacity } \\
\text { for medical decision making }\end{array}$ \\
\hline
\end{tabular}

*Some of these underemphasized competencies would be appropriately taught to all trainees

\section{Table 2. Guiding Principles for Developing Hospitalist-focused} Training

\section{Maintain balance}

Balance vocational needs with the needs of broadly trained generalists Complement rather than compete with other training interests Include strong core training in subspecialty and outpatient medicine Use existing ward rotations as a springboard for developing innovative clinical training opportunities

Build operational improvement methodology into clinical curricula Aim to develop expertise in both clinical and non-clinical realms Integrate non-clinical themes into the clinical rotations

Incorporate longitudinal reinforcement of key tenets

Utilize inpatient quality improvement and patient safety as the backbone of training

Require demonstration of ability to engage in and lead quality improvement efforts

\section{Emphasize process over clinical content}

Foster collegiality, respect and partnerships with primary care providers

Emphasize teamwork and multidisciplinary team approach

Enhance the communication aspects and safety of care transitions

\section{Keep the tent big}

Collaborate with other specialties to provide training venues

Include non-hospitalist faculty in the training program

Curricular elements should be available to all residents

ability to allocate hospitalist time to educational endeavors will depend in part upon who is funding the program. Successful hospitalist-training programs will need to align stakeholders' goals, or at least minimize conflicts early in the process.

Once mismatches have been identified and broad institutional support has been obtained, programs should match their local resources to their deficiencies. The paucity of skilled educators in underemphasized areas such as perioperative medicine, palliative care, $\mathrm{QI}$ and healthcare finance is a major barrier that will likely require faculty training, especially where curricula is being developed. This is an area where nonhospitalist educators can be enlisted to aid in the process. The challenge of educating future hospitalists may be compounded by the fact that academic hospitalists are generally junior faculty who are still developing their own skills as educators or content experts. Programs will need to nurture budding endemic talent and recruit where deficiencies still remain.

Next, programs will need to carve out dedicated rotations for hospitalist-track residents. As a first step, programs might expect residents to use elective time to fulfill their training needs. While this may be a reasonable "toe in the water" for newly forming programs, we believe that sustaining this approach sends a strong message that devalues these educational goals. We therefore recommend that hospitalist training

\section{Table 3. Framework for Developing Hospitalist-focused Training}

Perform local needs assessment to identify educational mismatches Generate institutional buy-in

Identify, develop and/or recruit educators with appropriate content expertise

Tie the training efforts to the clinical, quality and scholarly activities of faculty

Integrate curriculum longitudinally into clinical educational offerings Utilize existing training opportunities for developing hospitalist rotations Aim for comprehensive scope

Consider piloting clinical curricula first

Partner with other services to develop new clinical training opportunities Establish benchmarks for success and measure from the beginning 
supplant curricular activities that are deemed either overrepresented or less appropriate to hospitalist practice.

Accomplishing this task will frequently mandate shifting resident coverage away from other departmental services. Offsetting these staffing changes will require innovative staffing solutions, such as shifting clinical duties to hospitalists to free up housestaff. This could include substituting a hospitalist-run, non-resident service for a traditional inpatient ward team, which would free housestaff to rotate on a hospitalistspecific service. Additionally, housestaff could be replaced by non-physician practitioners on less educationally appropriate or overrepresented rotations. As an example, nurse practioners or physician assistants could cover bone marrow transplant units or cardiology services. Finally, some resident rotations can be modified to meet more than one core requirement. For example, shifting outpatient geriatric rotations to a more relevant inpatient setting can allow one rotation to fulfill both hospitalist and geriatric curricula requirements. All of these scenarios may require significant restructuring of services and reallocation of resources. Achieving success will require a clear vision, inspired leadership and strong institutional commitment to hospitalist-focused training.

We recommend aiming for a comprehensive hospital medicine curriculum for the latter part of IM training that covers the major areas of relative underemphasis. We recognize that many programs will take an incremental approach that initially focuses on a limited number of key curricular deficiencies. In our experience, it is easier to begin by targeting clinical deficiencies, such as perioperative medicine or palliative care, as more educators possess these skills, and it is often easier to get institutional and resident buy-in than for the often more abstract non-clinical areas.

Finally, programs must establish goals, evaluation methods and clearly defined benchmarks for success. The ability to document success and deliverables will be principal to the continued growth and success of the program.

\section{Summary}

Traditional categorical IM training may not meet the needs of many future internists, including hospitalists, who appear to be deficient in several core clinical and non-clinical areas of hospital medicine. As hospitalists rapidly become a fixture in the American medical landscape, it is increasingly vital to train them in the full spectrum of clinical and non-clinical hospital medicine. If we continue to matriculate hospitalists without addressing their specific training needs, we risk missing an opportunity to systemically improve the quality, safety and cost-effectiveness of inpatient care in the US.

*Drs. Glasheen, Prochazka, Kutner, Epstein and Siegal have no financial disclosures.

*All above-stated authors have contributed to the design, execution, analysis and writing of the following manuscript and will sign a document attesting to this. None of them have any conflicts of interest regarding this manuscript.

\section{Conflict of Interest: None disclosed.}

Corresponding Author: Jeffrey J. Glasheen, MD; Hospital Medicine Service, University of Colorado Denver School of Medicine, Mailstop F-782, 12401 E. 17th Avenue, P.O. Box 6510, Aurora, CO 80045, USA (e-mail: jeffrey.glasheen@uchsc.edu).

\section{REFERENCES}

1. Society of Hospital Medicine. Growth of Hospital Medicine Nationwide. Available at: http://www.hospitalmedicine.org/Content/NavigationMenu/ Media/GrowthofHospitalMedicineNationwide/Growth_of_Hospital_M.htm Accessed March 18, 2008.

2. Wachter RM. Reflections: The hospitalist movement a decade later. J Hosp Med. 2006; 1(4):248-52.

3. Society of Hospital Medicine. 2005-2006 SHM Survey: State of the Hospital Medicine Movement. Available at: http://dev.hospitalmedicine. org/AM/Template.cfm?Section=Survey\&Template=/CM/ContentDisplay. cfm\&ContentID=14352 Accessed March 18, 2008.

4. Plauth WH, Pantilat SZ, Wachter RM, et al. Hospitalist's perceptions of their residency training needs: Results of a national survey. Am J Med 2001;111:247-54.

5. Freese RB. The Park Nicollet experience in establishing a hospitalist system. Ann Intern Med. 1999;130:350-4.

6. Craig DE, Hartka L, Likosky WH, et al. Implementation of a hospitalist system in a large health maintenance organization: The Kaiser Permanente experience. Ann Intern Med. 1999;130:355-9.

7. Diamond HS, Goldberg E, Janosky JE. The effect of full-time faculty hospitalists on the efficiency of care at a community teaching hospital. Ann Intern Med. 1998;129:197-203.

8. Wachter RM, Katz $\mathbf{P}$, Showstack J, Bindman AB, Goldman L. Reorganizing an academic medical service. JAMA. 1998;279:1560-5.

9. Larson EB. Health care system chaos should spur innovation: Summary of a report of the Society of General Internal Medicine Task Force on the Domain of General Internal Medicine. Ann of Intern Med. 2004;140:639 43.

10. Fitzgibbons JP, Bordley DR, Berkowitz L, Miller BW, Henderson MC Redesigning residency training in internal medicine: A position paper from the Association of program directors in internal medicine. Ann Intern Med. 2006; 144:920-6.

11. Weinberger SE, Smith LG, Collier VU. Redesigning training for internal medicine. Ann Intern Med. 2006;144:927-32.

12. Auerbach AD, Wachter RM, Katz $\mathbf{P}$, et al. Implementation of a voluntary hospitalist service at a community teaching hospital: Improved clinical efficiency and patient outcomes. Ann Intern Med. 2002;137:85965.

13. Meltzer D, Manning W, Morrison J, et al. Effects of physician experience on costs and outcomes on an academic general medicine service: Results of a trial of hospitalists. Ann Intern Med. 2002;137:866-74.

14. Wachter RM, Goldman L. The hospitalist movement 5 years later JAMA. 2002;287:487-94

15. Sehgal NL, Wachter RM. The expanding role of hospitalists in the United States. Swiss Med Wkly. 2006;136(37-38):591-6.

16. Pham HH, Devers KJ, Kuo SK, Berenson R. Health care market trends and the evolution of hospitalist use and roles. J Gen Intern Med. 2005;20:101-7.

17. Vidyarthi AR, Arora V, Schnipper JL, Wall SD, Wachter RM. Managing discontinuity in academic medical centers: strategies for a safe and effective resident sign-out. J Hosp Med. 2006;1(4):257-66.

18. Cassel CK. Hospital Medicine: An important player in comprehensive care. J Hosp Med. 2006; 1:3-4.

19. Amin AN, Owen MM. Productive interdisciplinary team relationships: the hospitalist and the case manager. Lippincotts Case Manag. 2006;11 (3): $160-4$.

20. Cowan MJ, Shapiro M, Hays RD, Afifi A, Vaziarani S, Ward CR, Ettner SL. The effect of a multidisciplinary hospitalist/physician and advanced practice nurse collaboration on hospital costs. J Nurs Adm. 2006;36 (2):79-85

21. Ettner SL, Kotlerman J, Afifi A, Vazirani S, Hays RD, Shapiro M, Cowan M. An alternative approach to reducing the costs of patient care? A controlled trial of the multi-disciplinary doctor-nurse practitioner (MDNP) model. Med Decis Making. 2006;26(1):9-17.

22. Pantilat SZ, Rabow MW, Citko J, von Gunten CF, Auerbach AD, Ferris FD. Evaluating the California Hospital Initiative in Palliative Services. Arch Intern Med. 2006;166(2):227-30

23. Glasheen JJ, Epstein KR, Siegal E, Kutner J, Prochazka AV. The spectrum of community-based hospitalist practice, a call to tailor internal medicine residency training. Arch Intern Med. 2007;167 (7):727-8.

24. Huddleston JM, Long KH, Naessens JM, et al. Medical and surgical comanagement after elective hip and knee arthroplasty. Ann Intern Med. 2004;141:28-38. 
25. Phy MP, Vanness DJ, Melton LJ 3rd, et al. Effects of a hospitalist model on elderly patients with hip fracture. Arch Int Med. 2005; 165:796-801.

26. Roy A, Heckman MG, Roy V. Associations between the hospitalist model of care and quality-of-care-related outcomes in patients undergoing hip fracture surgery. Mayo Clin Pro. 2006;81(1):28-31.

27. Ovbiagele B, Sachdeva S. Preventing another event: Role of the hospitalist in discharge stroke prevention. J Hosp Med. 2007;2:31-8.

28. Salerno SM, Hurst FP, Halvorson S, Mercado DL. Principles of effective consultation: An update for the 21st-century consultant. Arch Intern Med. 2007; 167:271-5

29. Jaffer AK, Brotman DJ, Sridharan ST, et al. Postoperative pulmonary complications: experience with an outpatient preoperative assessment program. J Clin Outcomes Manage. 2005;12(10):505-10. Oct.

30. Kearns PJ, Wang CC, Morris WJ, et al. Hospital care by hospital-based and clinic-based faculty. Arch Intern Med. 2001;161:235-41.

31. Kaboli PJ, Barnett MJ, Rosenthal GE. Associations with reduced length of stay and costs on an academic hospitalist service. Am J Manag Care. 2004; 10:561-8.
32. Rifkin WD, Holmboe E, Scherer H, Sierra H. Comparison of hospitalists and nonhospitalists in inpatient length of stay adjusting for patient and physician characteristics. J Gen Intern Med. 2004;19: 1127-32.

33. Halasyamani LK, Valenstein PN, Friedlander MP, Cowen ME. A comparison of two hospitalist models with traditional care in a community teaching hospital. Am J Med. 2005;118:536-43.

34. Accreditation Council for Graduate Medical Education Core Competencies. Available at http://www.acgme.org/outcome/comp/compMin. asp Accessed March 17, 2008.

35. Pistoria MJ, Amin AN, Dressler DD, et al. The core competencies in hospital medicine. J Hosp Med. 2006; 1(S1):1-95.

36. McKean SC, Budnitz TL, Dressler DD, et al. How to use the core competencies in hospital medicine: A framework for curriculum development. J Hosp Med. 2006;1:57-67.

37. Ranji SR, Rosenman DJ, Amin AN, Kripalani, S. Hospital medicine fellowships: works in progress. Am J Med. $2006 ; 119$ (1):72.e1-7.

38. Hauer KE, Flanders SA, Wachter RM. Training future hospitalists. West J Med. 1999;171:367-70. 